\title{
ASSESSMENT OF MAJOR AND MINOR HAEMORRHAGE IN A HIGH RISK NEONATAL POPULATION USING A VALIDATED STRUCTURED BLEEDING ASSESSMENT TOOL
}

\author{
V. Venkatesh ${ }^{1}$, R. Khan ${ }^{1}$, A. Curley ${ }^{1}$, P. Clarke ${ }^{2}$, P. Muthukumar ${ }^{2}$, T. Watts ${ }^{3}$, H. New ${ }^{4}$, F. Sweeney ${ }^{5}$, S. \\ Stanworth ${ }^{6}$, Platelets for Neonatal Transfusion (PlaNet) Study Group \\ ${ }^{1}$ NICU, Cambridge University Hospitals NHS Foundation Trust, Cambridge, ${ }^{2}$ NICU, Norfolk \& Norwich \\ University Hospitals NHS Trust, Norwich, ${ }^{3}$ NICU, St Thomas' Hospital, ${ }^{4}$ Haematology, St Mary's Hospital at \\ Imperial College, London, ${ }^{5}$ NHSBT, NHSBT, Bristol, ${ }^{6}$ Haematology, NHSBT, Oxford, UK
}

Background: Infants admitted to neonatal intensive care are at high risk of bleeding. Our objective was to develop and pilot a bleeding assessment tool (BAT) and validate its use in neonates admitted to intensive care

Methods: The BAT was developed, reviewed and modified from the WHO bleeding score by a team of neonatologists, haematologists and statisticians. It was used as a 14 day prospective daily record of bleeding to assess incidence of all bleeding in neonates admitted for high dependency/intensive care in 6 tertiary NICUs over 2-4 week admission period. Independent personnel performed duplicate assessmentsin order to standardise and validate the tool.

Results: 74 neonates were assessed for 648 days. Median birth weight:1725 (IQR 890-3380)g, median gestational age: 30 (IQR 26-38) weeks. 30/74 (41\%) babies developed some form of bleeding. Incidence of bleeding (represented as bleeding days/study days) was: severe 48/648 (7.4\%); major 74/648 (11.5\%); moderate 21/648 (3.2\%); minor 183/648 (28.2\%). Babies born at $<28$ weeks gestation had a greater incidence of both overall bleeding ( $68 \%$ vs $27 \% \mathrm{p}<0.05)$ and major/severe bleeding ( $30 \%$ vs $10 \% \mathrm{p}<0.05)$. Major bleeds included: intraventricular haemorrhage (12\%), rectal haemorrhage (6\%); pulmonary haemorrhage (7\%). 218 of 648 daily assessments were duplicated. $86 \%$ of duplicate records matched (p< $0.001)$.

Conclusions: One in five babies in this study experienced major or severe bleeds. Minor bleeding is common although its significance remains unclear. Interventions to reduce bleeding in neonates will benefit from a validated recording system such as this BAT developed specifically for use in neonates. 